\title{
L'étain et les organoétains dans l'environnement
}

\section{Tin and organotins in the environment}

Jean-Pierre ANGER

Laboratoire de Toxicologie, Faculté de Pharmacie, 2, avenue du Professeur Léon Bernard 35043 RENNES Cedex - Tél : 33 (0)2 99336936 - Fax : 33 (0)2 99336242

(Reçu le 15 février 2001 ; accepté le 20 mars 2001)

\section{RÉSUMÉ}

L'étain sous forme minérale ou organique connaît de nombreuses applications industrielles. Sa présence dans l'environnement est essentiellement d'origine anthropogénique. Toutes les formes minérales et organiques de l'étain y sont présentes aussi bien les produits issus de la biodégradation des organoétains que ceux résultant de la biométhylation de l'étain minéral. Si les sels minéraux de l'étain sont considérés comme peu toxiques pour les mammifères, il n'en est pas de même pour les composés organiques qui interférent avec de nombreux mécanismes biochimiques intracellulaires. L'écotoxicologie de l'étain et des organoétains est une préoccupation relativement récente liée essentiellement à la dispersion des dérivés trialkylés et triarylés dans l'environnement aquatique par suite de leur utilisation dans les peintures marines "antisalissures » ou en agriculture. Ces composés, fortement lipophiles, sont susceptibles de s'accumuler dans les chaînes alimentaires. L'examen des données disponibles pour les animaux aquatiques montre des effets significatifs sur la croissance et la reproduction des organismes phyto et zooplanctoniques pour des concentrations dans l'eau inférieures au $\mu g / l$. Il ne semble pourtant pas qu'il y ait actuellement des risques particuliers pour la santé humaine par le biais de l'ingestion de crustacés ou de poissons contaminés. Des dispositions législatives ont été prises interdisant notamment l'utilisation des peintures marines antisalissures pour les bateaux de longueur inférieure à 25 mètres. Elles impliquent donc une surveillance analytique basée sur la spéciation de l'étain après extraction du milieu, dérivation et concentration, grâce aux méthodes chromatographiques séparatives couplées aux systèmes de détection modernes (spectrométrie de masse, émission atomique).

\section{SUMMARY}

Tin in the mineral or organic form knows many industrial applications. Its presence in the environment is essentially of anthropogenic origin. All forms of mineral and organic tin are present as well stemming products of the organotin biodegradation as those resulting from the biomethylation of mineral tin. If mineral salts of tin are considered as poorly toxic for mammals, it is not the same for organic compounds that interfere with many biochemical intracellular mechanisms. Tin and organotin ecotoxicology is a relatively recent preoccupation linked essentially to the dispersion of the trialkyl and triaryl derivatives in the aquatic environment as a result of their utilization in marine antifouling paints or in agriculture. These strongly lipophilic compounds are susceptible to accumulate in food chains. Available data for aquatic animals show significant effects on the growth and the reproduction of phyto and zooplanktonic organisms, for water levels less than $1 \mu \mathrm{g} / \mathrm{l}$. Nevertheless it does not seem that there are currently particular risks for the human health when ingesting crustaceans or contaminated fish. Legislative dispositions have been taken forbiding notably antifouling paints for inferior length boats to 25 meters. They imply therefore an analytic supervision based on the speciation of tin after extraction of the environmental media, derivation and concentration, thanks to chromatographic methods coupled to modern detection systems (mass spectrometry, atomic emission spectroscopy). 


\section{Sources d'entrées de l'étain dans l'environnement}

L'étain fait partie de la colonne IV b de la classification périodique des éléments au même titre que le carbone, le silicium, le germanium et le plomb. Ce métal, inaltérable à l'air, présente la valence +2 ou +4 .

L'étain métal et ses sels divalents (généralement minéraux) possèdent des applications industrielles nombreuses : étamage des métaux, fabrication du fer blanc, soudures et alliages divers, catalyseurs en synthèse organique, industrie des céramiques, etc.

À l'état tétravalent, l'étain se lie au carbone pour donner des composés appelés organoétains ou organostanniques. Ces composés dans lesquels 1 à 4 atomes de carbone peuvent être liés directement à l'atome d'étain répondent à la formule générale $\mathrm{R} n \mathrm{Sn} \mathrm{X}(4-n)$ où $n$ varie de 1 à $4, \mathrm{R}$ est un groupement organique aliphatique ou aromatique et $\mathrm{X}$ une espèce ionique (halogénure, carbonate, oxyde, etc.). Ce sont pour la plupart des produits incolores, liquides ou facilement fusibles, très solubles dans les solvants organiques et les huiles et assez volatils. Leur solubilité dans l'eau est le plus souvent faible.

La production des dérivés organiques de l'étain qui était pratiquement nulle dans les années $50 \mathrm{~s}$ 'est considérablement accrue depuis. Elle est estimée aujourd'hui à environ 40000 tonnes annuelles (1).

Hormis les tétraorganoétains $\left(\mathrm{R}_{4} \mathrm{Sn}\right)$ qui restent d'importants intermédiaires dans la fabrication des autres composés organostanniques, seuls les monoorganoétains $\left(\mathrm{RSnX}_{3}\right)$, les diorganoétains $\left(\mathrm{R}_{2} \mathrm{SnX} \mathrm{X}_{2}\right)$ et les triorganoétains $\left(\mathrm{R}_{3} \mathrm{SnX}\right)$ ont trouvé des applications variées dans les secteurs industriel, agronomique et pharmaceutique.

Rappelons qu'en France, en 1953, le diiododiéthylétain dont l'action anti-staphylococcique avait été démontrée expérimentalement quelques années plus tôt fut proposé dans le traitement par voie orale de la furonculose, sous forme de capsules (STALINON ${ }^{\circledR}$ ): Malheureusement ce produit contaminé par $10 \%$ environ d'iodure de triéthylétain provoqua un grand nombre d'intoxications dont certaines furent mortelles (102 morts sur 210 cas rapportés). Ces accidents entrấnèrent une méfiance tout à fait justifiée des thérapeutes, médecins et vétérinaires envers tous les médicaments pouvant contenir des dérivés de l'étain sans distinction. Aujourd' hui le domaine d'application le plus important des composés organoétains est la stabilisation du chlorure de polyvinyle (PVC). La stabilisation évite dans une large mesure la coloration du polymère lors de sa transformation à haute température ou sa dégradation sous l'action ultérieure de la lumière. Les composés les plus utilisés dans ce domaine sont les esters de dibutyl ou de dioctylétain.

Les composés trisubstitués possèdent des propriétés fortement biocides et à cet effet sont utilisés dans diverses circonstances :

- dans la préparation de peintures marines antisalissures («antifouling paints»). Ces peintures sont destinées à lutter contre la prolifération des animaux et plantes marines sur les coques des bateaux, ce qui ralentit leur marche. Les produits les plus utilisés dans ce but sont l'oxyde de tributylétain, le chlorure et le fluorure de triphénylétain ;

- dans la préservation du bois, des textiles et des vêtements contre les attaques cryptogamiques et bactériennes qui décomposent à la longue la cellulose et les protéines ;

- dans la protection des récoltes, en milieu agricole, certains dérivés du triphénylétain se sont révélés très efficaces contre une large gamme de maladies dues à la pourriture qui attaque les céréales essentielles.

D'autres utilisations commerciales intéressantes mais de moindre importance peuvent être citées : désinfectants en milieu hospitalier, inhibiteurs de la formation de mucilage microbien dans l'industrie papetière, agents répulsifs contre les rongeurs, acaricides, etc.

La consommation annuelle française en organoétains serait actuellement de $3000 \mathrm{~T}$.

Toutes ces utilisations sont responsables de la présence en quantité non négligeable de l'étain sous forme minérale ou organique dans l'environnement et posent de ce fait un problème de santé publique car, si les sels minéraux de l'étain sont considérés comme relativement peu toxiques, il n'en est pas de même pour les dérivés organiques qui possèdent une toxicité élevée aussi bien pour les mammifères que pour les espèces aquatiques.

Les principales sources d'entrée de l'étain dans l'environnement peuvent se résumer ainsi :

- les traitements phytosanitaires, utilisant principalement les dérivés triphénylétains appliqués par dispersion aérienne ont pour conséquence la contamination de l'atmosphère par les aérosols, celle des sols par dépôt et celle des eaux de surface, par lixiviation des sols ;

- l'emploi des peintures antisalissures a pour résultat l'introduction de tributylétain à la fois dans le milieu marin et dans l'atmosphère lors des opérations de carénage des navires ;

- le relargage des di et des monoalkylétains à partir des emballages à base de PVC peut contaminer les eaux usées et ultérieurement l'environnement aquatique ;

- il semble heureusement que l'incinération des déchets domestiques ne soit pas en mesure d'introdui- 
re des quantités notables d'organoétains dans l'atmosphère, compte tenu de leur instabilité thermique et de leur transformation ultime en oxyde stannique $\mathrm{SnO}_{2}$.

\section{Distribution et devenir de l'étain dans l'environne- ment. Niveaux de contami- nation}

Ce n'est qu'à partir des années 80 que les techniques analytiques de spéciation ont permis la mise en évidence des différentes formes d'étain présentes dans l'environnement.

\section{Étain minéral}

En milieu aquatique, l'étain inorganique peut se trouver sous forme dissoute et semble-t-il, de façon plus importante, sous forme particulaire comme l'ont montré les résultats obtenus par BYRD ET ANDREAE (2) sur les eaux d'une quarantaine de rivières. Les formes chimiques de l'étain dissous sont encore mal connues et correspondent sans doute aux états d'oxydation II ou IV. Il est par ailleurs bien établi que l'étain inorganique peut être méthylé en milieu aquatique par voie biotique ou abiotique tout comme d'autres métaux et métalloïdes tels l'arsenic, le mercure ou le plomb. Des donneurs de méthyle : méthylcobalamine, bétaïne, méthylméthionine, etc. ont d'ailleurs été identifiés dans l'environnement (3)

En eau douce, les teneurs en étain minéral sont généralement de l'ordre du $\mathrm{ng} / \mathrm{l}$, mais peuvent atteindre le $\mu \mathrm{g} / \mathrm{l}$ dans certaines zones polluées. Dans les océans, les concentrations varient de quelques dixièmes de $\mathrm{ng} / \mathrm{l}$ au large à quelques ng/l à la côte. On ne dispose pratiquement pas de valeurs pour les sédiments et les organismes marins car les résultats sont le plus souvent exprimés en étain total.

\section{Étain organique}

Le comportement géochimique des organoétains a surtout été étudié pour les tributyl (TBT) et triphénylétains (TPT) du fait de leur utilisation prépondérante dans cette série. Dans l'eau de mer, le TBT coexiste ainsi sous trois formes : chlorure, hydroxyde et carbonate, essentiellement à l'état dissous. Les sédiments pourraient jouer un rôle de réservoir avec échange ultérieur avec le milieu aquatique.

La liaison carbone-étain étant un point de moindre résistance dans la molécule d'organoétain, leur dégradation par déalkylations successives est relativement facile. La dégradation par photolyse UV des tri, di et monoorganoétains a été expérimentalement démontrée
(4). Dans tous les cas elle aboutit à la formation d'oxyde stannique. La dégradation des tributyl et triméthylétains en milieu aqueux est cependant plus rapide que celle des dérivés disubstitués et les mécanismes réactionnels sont encore mal connus. La demi-vie du TBT dans l'eau varie en fonction des conditions de milieu, de $\mathrm{pH}$, de température, de turbidité et d'éclairement (5). La dégradation est plus rapide dans les eaux de mer chargées de matières en suspension. La persistance du TBT est par contre très élevée dans les sédiments où sa demi-vie est estimée de quelques années à quelques dizaines d'années.

\section{Niveaux de contamination des eaux et des sédiments}

La contamination des milieux marins par le TBT est connue depuis longtemps. Elle est directement reliée aux activités de plaisance et de commerce maritime. Dans les années 80 , on a pu retrouver dans l'eau de mer des marinas des teneurs allant de 100 à $500 \mathrm{ng} / \mathrm{l}$ de TBT . Ces valeurs étaient nettement plus élevées que celles des eaux de surface dans les baies et les estuaires où les concentrations observées se révélèrent dix fois plus faibles. Les teneurs étaient plus fortes en été mais dépendaient considérablement des mouvements de la marée. Cette contamination a nettement diminué depuis dans les pays où il existe une réglementation concernant l'usage des peintures antisalissures. Lorsque cette dernière est absente, on en retrouve des teneurs importantes comme c'est le cas dans le fond du golfe Persique ou à Hong Kong où des concentrations de 14,7 et $1 \mu \mathrm{g} / \mathrm{l}$ de TBT ont pu être respectivement mesurées (6). Étant donné la longue demi-vie de dégradation du TBT dans les sédiments, il n'est pas surprenant de constater sa présence en quantité importante dans les aires portuaires et les baies. On a pu observer ainsi au Japon des concentrations allant jusqu'à $2,7 \mathrm{mg} / \mathrm{kg}$ (poids sec) de sédiment dans les sites les plus contaminés.

La contamination des rivières et des lacs est un peu moins connue. Les suivis réalisés par divers auteurs dans les grands lacs d'Amérique du Nord ont permis de détecter la présence de butyl et de méthylétains mono, di et trisubstitués. Les concentrations maximales sont de l'ordre de $10 \mu \mathrm{g} / \mathrm{l}$ dans les eaux de surface. Entre 1983 et 1990 des études. réalisées sur différents lacs suisses montrent des teneurs en TBT allant de 0,001 à $1,64 \mu \mathrm{g} / \mathrm{l}$, les concentrations les plus élevées se retrouvant dans les ports. Il a même été détecté du TPT, sans doute d'origine agricole. Dans les sédiments lacustres, la pollution par le TBT dépend de la profondeur et se situe en moyenne autour de 0,5 à $2,5 \mathrm{mg} / \mathrm{kg}$ (poids sec) (6).

En ce qui concerne le littoral français, les résultats d'un suivi réalisé en 86 et 87 sur la côte atlantique indi- 
quaient des teneurs en TBT comprises entre 2 et $50 \mathrm{ng} / \mathrm{l}$ dans les eaux côtières, 10 à $150 \mathrm{ng} / \mathrm{l}$ (exceptionnellement $1500 \mathrm{ng} / \mathrm{l}$ ) dans celles des ports de plaisance (7) et jusqu'à $1,48 \mathrm{mg} / \mathrm{kg}$ dans les sédiments de la baie d'Arcachon (8). Sur la côte méditerranéenne, un échantillonnage réalisé en 88 indiquait des teneurs maximales n'excédant pas $600 \mathrm{ng} / \mathrm{l}$ dans les ports de plaisance, $200 \mathrm{ng} / \mathrm{l}$ dans un port de commerce et de l'ordre de 2 ng/l dans l'étang de Thau (9).

Ainsi toutes les formes minérales et organiques de l'étain peuvent se retrouver dans l'environnement qu'il s'agisse des produits de biodégradation des organoétains ou des produits résultant de la bio méthylation de l'étain minéral.

\section{Bioconcentration de l'étain dans les organismes aqua- tiques}

L'étain minéral ne semble pas s'accumuler dans les organismes vivants. Il n'en est pas de même pour le TBT, composé à caractère lipophile qui est capable de se concentrer dans les bactéries (10), le phytoplancton (11), les mollusques, notamment l'huître (12), la coquille Saint Jacques (13) et la moule (11). Le même phénomène a été observé chez les crustacés, notamment le crabe (14) lorsque la contamination se fait par l'intermédiaire de la nourriture et le poisson, tout spécialement chez les salmonidés élévés dans des cages traitées par des peintures antisalissures à base de TBT $(15,16)$. Les facteurs de bioconcentration varient dans de très grandes proportions de 1 à 30000 sans qu'il soit possible de déterminer une fourchette de valeurs par groupes d'espèces (1).

\section{Risques pour le consommateur}

Plusieurs travaux ont montré l'existence de résidus d'organoétains dans les organismes aquatiques. Qu'en est-il des risques liés à la consommation des produits de la mer pour la santé humaine ?

MAGUIRE et àl. (17) ont retrouvé jusqu'à $0,49 \mathrm{mg} / \mathrm{kg}$ de TBT dans le poisson entier ainsi que du mono et du diméthylétain. On a également détecté des taux importants de TBT dans les poissons élevés en aquaculture, par exemple de 0,4 à $3,1 \mathrm{mg} / \mathrm{kg}$ dans le saumon d'Écosse (16). La cuisson ne détruit pas et n'élimine pas le TBT si bien que l'organoétain peut entrer dans le régime alimentaire de l'homme. Au Japon, la présence de TBT et de TPT dans les produits de la mer est également bien connue. On en retrouve des teneurs allant de 0,02 à $1,3 \mathrm{mg} / \mathrm{kg}$, notamment chez le maquereau (18), surtout dans le foie et les reins, plus que dans le tissu musculaire. Les moules, les palourdes et les calamars contiennent de 10 à $100 \mathrm{mg} / \mathrm{kg}$ de TBT.

Tous ces résultats démontrent bien que l'homme peut se trouver exposé au TBT et au TPT via le poisson, les mollusques et autres produits de la mer. Les taux présents cependant ne sont pas considérés comme dangereux pour la santé humaine car ils restent bien en dessous de la dose journalière acceptable (DJA). Pour l'adulte, en effet, une DJA provisoire de 3,2 $\mu \mathrm{g}$ de TBT $/ \mathrm{kg}$ de poids corporel a été proposée en 1987 par SCHWEINFURTH et GUNZEL (19), soit $192 \mu \mathrm{g}$ pour un sujet de $60 \mathrm{~kg}$. À titre d'exemple, la dose sans effet (D.S.E) de l'oxyde de tributylétain dans le régime alimentaire chez le rat est comprise entre 0,5 et $5 \mathrm{mg} / \mathrm{kg}$ de nourriture soit entre 0,025 et $0,25 \mathrm{mg} / \mathrm{kg}$ de poids corporel (6). Comme la consommation de poisson ou de coquillage est très variable chez l'homme et que les résidus d'organoétains sont également très variables, on ne peut faire que de grossières approximations. En supposant donc une consommation quotidienne de $150 \mathrm{~g}$ de poisson avec un taux résiduel de TBT de 1 $\mathrm{mg} / \mathrm{kg}$, le sujet ingèrerait $150 \mu \mathrm{g}$ de TBT, soit $2,5 \mu \mathrm{g} / \mathrm{kg}$ pour un sujet de $60 \mathrm{~kg}$. Ceci correspondrait donc par rapport à la DSE chez le rat à un facteur de sécurité de 10 à 100 ce qui reste tout à fait raisonnable.

\section{Mécanismes d'action des organoétains}

La toxicologie des composés organostanniques peut être résumée brièvement comme suit : les effets biologiques des tétraorganoétains chez les mammifères semblent être entièrement dus à leur conversion, in vivo, au niveau hépatique, en dérivés trisubstitués correspondants. Dans la série des composés trialkylétains, les composés triéthylés sont les plus toxiques pour les mammifères. Si la longueur de la chaîne du groupement alkyl augmente, l'activité biologique diminue de telle sorte que les composés trioctylétains sont pratiquement dépourvus de toxicité pour toutes les espèces vivantes. Les composés triarylétains apparaissent aussi toxiques que les tributylétains. L'anion sous lequel se présente le composé trisubstitué n'a, en général, que très peu d'importance sur l'activité. Les composés dialkylétains voient également leur toxicité diminuer avec l'allongement des chaînes alkyles. Les monoorganoétains ne semblent pas présenter de toxicité importante à l'égard des mammifères.

Il résulte donc de ce bref aperçu que seuls, les di et les triorganoétains sont capables d'interagir avec les mécanismes biochimiques intracellulaires et surtout les composés trisubstitués. 
Les composés tributylétains perturbent la production énergétique cellulaire. Ils inhibent à doses très faibles (inférieures à $1 \mu \mathrm{M}$ ) la phosphorylation oxydative dans une très grande variété de cellules et découplent la photo phosphorylation dans les chloroplastes (20). Ils endommagent les membranes plasmiques et inhibent les pompes ioniques. Il semble que le point de départ de ces actions soit dû à une altération de l'homéostasie calcique intracellulaire, notamment une augmentation du calcium cytosolique. Ce mécanisme est également à l'origine de l'induction de l'apoptose des thymocytes ce qui explique les propriétés immunotoxiques du TBT (21). Les protéines intracellulaires sont également dégradées par suite de leur interaction entre l'organoétain et leurs acides aminés constitutifs notamment la cystéine et l'histidine (22).

\section{Toxicité du tributylétain à l'égard des espèces aqua- tiques}

L'écotoxicologie des organoétains est une préoccupation relativement récente liée essentiellement à la dispersion des trialkylétains dans l'environnement aquatique. Les propriétés inhibitrices vis à vis de la phosphorylation oxydative sont responsables d'effets toxiques à l'égard des différents embranchements zoologiques. L'examen des données disponibles pour les animaux aquatiques montre que les effets significatifs sur la croissance et la reproduction d'organismes, phyto et zoo planctoniques ont été obtenus pour des concentrations en TBT inférieures au $\mu \mathrm{g} / \mathrm{l}$. Les mollusques sont particulièrement sensibles. À la concentration de $0,2 \mu \mathrm{g} / \mathrm{l}$, la survie des huîtres est affectée au bout de deux mois tandis que le seuil de survie à long terme des bivalves adultes serait voisin de $0,04 \mu \mathrm{g} / \mathrm{l}$. Ces seuils correspondent à des niveaux retrouvés dans les milieux naturels situés à proximité des zones d'apports en TBT. Les résultats des expérimentations en laboratoire, confirmés par les observations en milieu naturel apportent la preuve que le TBT, à des concentrations inférieures au ng/l, se comporte comme un « disrupteur hormonal » en modifiant de façon significative les caractères sexuels des gastéropodes marins. Les effets observés consistent en l'apparition d'organes génitaux mâles chez les femelles ou " imposex ». Le développement de l'imposex chez les gastéropodes femelles, le signe le plus sensible de l'écotoxicité du TBT n'est pas un effet direct, mais est médié par les hormones stéroïdiennes. Le TBT serait responsable d'une augmentation de la testostérone par suite de l'inhibition de la cyt $\mathrm{P} 450$-aromatase responsable de la conversion de la tes- tostérone en $17 \beta$ œstradiol (23). On ne sait pas si l'enzyme est détruite ou inhibée par le TBT.

L'influence du TBT sur la calcification des coquilles de l'huître Crassostrea gigas a été prouvée à la fois in situ et au laboratoire. Elle se traduit par l'apparition d'un gel inter lamellaire perturbant la formation de la coquille et ce, à partir de concentrations dans l'eau de mer, aussi faibles que $2 \mathrm{ng} / \mathrm{l}$.

Chez les poissons, l'un des points surprenants de la toxicité des dérivés trisubstitués consiste en l'inhibition spécifique de certains cytochromes $\mathrm{P} 450$ des hépatocytes. Plusieurs constituants de ces systèmes fondamentaux de détoxication sont touchés : à la fois l'hémoprotéine et l'activité enzymatique ainsi que les NADPH-CytP450 réductases. Les triorganoétains (tributyl et triphényl) agissent par des mécanismes directs et non par un mécanisme suicidaire lié à la formation d'un métabolite toxique. Les Cyt P450 1 A qui sont induits par de nombreux xénobiotiques environnementaux semblent être sélectivement affectés chez le poisson à la fois in vivo et in vitro. D'autres isoformes du Cyt-P450, dont celle qui est responsable de l'hydroxylation de la testostérone, sont également affectées, mais pour des concentrations supérieures à $10 \mu \mathrm{M}(6)$. La toxicité aiguë des triorganoétains pour le poisson est d'ailleurs bien illustrée par la cytotoxicité in vitro déterminée sur les hépatocytes de vairon : la $\mathrm{C}$ I 50 pour les hépatocytes de Poeciliopsis lucida est de 0,1 et $0,7 \mu \mathrm{M}$ respectivement pour le TBT et le TPT. Cette cytotoxicité est liée à la fois à la lipophilie des organoétains et à leur toxicité aiguë in vivo. C'est pour cette raison que les essais in vitro réalisés sur des hépatocytes de poisson en culture peuvent aujourd'hui constituer des outils valables pour évaluer la toxicité des agents chimiques environnementaux.

\section{Analyse des organoétains dans l'environnement}

Les organoétains présents dans l'environnement aquatique se retrouvent le plus souvent associés à une grande variété d'anions (carbonates, chlorures, sulfate, hydroxydes, etc.). Ils interagissent avec les matrices abiotiques par des liaisons ioniques et/ou hydrophobiques. Ils devront donc être extraits de la matrice avant d'être séparés puis analysés à l'aide de détecteurs spécifiques.

L'analyse se déroule en plusieurs étapes que l'on peut résumer comme suit (24):

\section{Première étape : Extraction}

Cette étape est habituellement exécutée sur des échantillons desséchés par cryodessiccation, évaporation ou 
par voie chimique. L'extraction s'effectue à l'aide de solvants apolaires (hexane, toluène, dichlorométhane, etc), par agitation ou sonication. Cette opération est effectuée après acidification du milieu ou digestion acide afin de relarguer les organoétains de la matrice. On peut recourir également à des solvants polaires (méthanol, acétone, acétate d'éthyle). Quelques techniques font appel, dans le cas des sédiments à l'extraction en phase supercritique. Pour améliorer l'extraction des dérivés mono et disubstitués, on peut utiliser des agents complexants de l'étain comme la tropolone ou le diéthyldithiocarbamate d'ammonium.

\section{Deuxième étape : Dérivation}

Les analyses chromatographiques en phase gazeuse (C.P.G) nécessitent souvent une réaction de dérivation pour produire des organoétains volatils afin d'améliorer leur séparation. Deux méthodes sont principalement utilisées :

- l'alkylation par méthylation, éthylation, propylation, pentylation ou hexylation suivie d'une extraction liquide-liquide. On peut également faire appel au tétraéthylborate de sodium qui s'adapte plus particulièrement aux échantillons aqueux, en milieu tamponné.

- la formation d'hydrures avec le borohydrure de sodium qui permet l'analyse des butylétains et des organoétains très volatils comme le méthylétain ou encore les phénylétains.

\section{Troisième étape : Purification de l'extrait}

La plupart des techniques analytiques utilisant la C.P.G nécessite une étape de purification généralement après l'étape de dérivation. La silice est l'adsorbant le plus souvent cité mais également l'alumine, le florisil et les cartouches $\mathrm{C}_{18}$. Le florisil est particulièrement bien adapté aux matrices biologiques à forte teneur en lipides. L'hexane ou le mélange hexane-éther éthylique sont très souvent utilisés comme éluants car ils permettent ensuite l'analyse directe par C.P.G sans évaporation à sec.

\section{Quatrième étape : Dosage proprement dit}

Dans la plupart des méthodes, les organoétains sont séparés par chromatographie avant leur détection. La C.P.G et la chromatographie liquide haute performance (C.L.H.P) sont les techniques les plus souvent citées dans la littérature.

En C.P.G, les colonnes remplies ont permis dans les années 80 , la séparation des organoétains. Aujourd'hui ces colonnes ne sont guère plus utilisées que pour piéger à très basse température, les hydrures volatils qui seront ensuite désorbés par un chauffage rapide. Dans les années 90, l'utilisation des colonnes capillaires a permis une meilleure résolution et une meilleure détec- tion des composés élués. Les phases non polaires (DB 1, SE 30, HP 5, etc. ) ou moyennement polaires (OV 17) conviennent parfaitement. La détection des organoétains est réalisée par ionisation de flamme, capture d'électrons, spectrométrie d'absorption atomique (S.A.A), photométrie de flamme, émission atomique (E.A) ou spectrométrie de masse (MS). L'I.C.P-MS reste évidemment le moyen de détection le plus sensible et le plus spécifique.

Les applications de la C.H.L.P à la spéciation de l'étain dans l'eau ou les matrices biologiques sont beaucoup moins nombreuses. Cela tient sans doute à la sensibilité insuffisante de la plupart des détecteurs comparée aux taux retrouvés dans les échantillons biologiques. Parmi les techniques proposées, la chromatographie ionique sur colonne échangeuse cationique a été particulièrement développée. Avec une phase mobile à base de méthanol ou parfois un mélange acétonitrile-eau en milieu tamponné citrate ou acétate, on peut séparer les butylétains. De nombreux types de détection ont été appliqués à l'analyse chromatographique des organoétains : S.A.A, I.C.P-M.S, fluorimétrie, etc.

De façon générale, la sensibilité de ces techniques dépend essentiellement du type de détecteur utilisé. Elles permettent cependant d'atteindre les niveaux de la ppb et parfois de la ppt selon la matrice, concentrations auxquelles les organoétains sont susceptibles de perturber le milieu environnemental.

\section{Conclusion}

Le bilan des effets écotoxicologiques des organoétains, notamment les composés tributylés vis-à-vis de l'environnement aquatique est loin d'avoir été complètement évalué. C'est sans doute en vertu du principe de précaution que la France fut dès 1982, le premier état à interdire l'utilisation des peintures contenant des organoétains sur tous les bateaux d'une longueur inférieure à 25 mètres. Cette disposition a été suivie par le Royaume-Uni et les Etats-unis, en 1987 puis adoptée au plan international en 1990. Comme il a été démontré par la suite, pour le bassin d'Arcachon, l'application de cette réglementation a permis une amélioration très nette de la qualité de l'eau et s'est accompagnée d'une restauration progressive de l'activité ostréicole (25). On doit cependant remarquer que l'interdiction d'emploi du TBT a conduit les usagers à utiliser des peintures dont la matière active est de l'oxyde de cuivre, ce qui a eu pour conséquence d'élever le niveau de la présence de cet oligo-élément dans les eaux et les chairs d'huîtres (26). Affaire à suivre, peut-être ! 


\section{Références}

1. Alzieu C., Michel P. L'étain et les organoétains en milieu marin : biogéochimie et écotoxicologie, Ed IFREMER, 1998, $104 \mathrm{p}$.

2. Byrd J. T., Andreae M.O. Geochemistry of tin in rivers and estuaries, Geochim Cosmochim Acta, 1986a, 50 : 835-845.

3. Craig P.J., Rapsomanikis S. Methylation of tin and lead in the environment : oxydative methyl transfer as a model for environmental reactions. Environ. Sci. Technol., 1985, $19:$ 726-730.

4. Blunden S.J., Chapman A.H. The environmental degradation of organotin coumpounds. A review. Environ. Technol. Lett., 1983, $3: 267-272$.

5. Maguire R.J., Carey J.H., Hale E.J. Degradation of the tri-n-butyl species in water. J. Agric. Food Chem., 1983, $31: 1060-1065$.

6. Fent K. Ecotoxicology of organotin coumpounds, Critical Reviews in Toxicology, 1996, 26(1) : 1-117.

7. Alzieu C., Sanjuan J., Michel P., Borel M., Dreno J.P. Monitoring and assessment of butyltins in atlantic coastal waters, Mar. Pollut. Bull., 1989, 20(1) : 22-26.

8. Saradin P.M., Astruc A., Sabrier R., Astruc M. Survey of butyltin coumpounds in Arcachon bay sediments, Mar. Pollut. Bull., 1994, 28 : 621-26.

9. Alzieu C., Michel P., Sanjuan J., Averty B. Tributyltin in french mediterranean coastal waters, Appl. Organomet. Chem, 1990, $4: 55-61$.

10. Blair W. R., Olson G.J., Trout T.K., Jewett K.L., Brinckman F.E. Accumulation and fate of tributyltin species in microbial biofilms, in Ocean's 88 Conference Proceedings. Organotin Symposium, 1988, $4:$ 16681672.

11. Laughlin R.B., French W., Guard H.E. Accumulation of bis tributyltin oxide by the marine mussel Mytilus edulis, Environ. Sci. Technol., 1986, 20, $9: 884-890$.

12. Waldock M.J., Thain J.E., Miller D. The accumulation and depuration of bis tributyltin oxide in oysters : a comparaison between the pacific oyster Crassostrea gigas and the european flat oyster Ostrea edulis, I.C.E.S, CM, 1983/E : 59-64.

13. Davies I. M., Mc Kie J.C., Paul J.D. Accumulation of tin and tributyltin from antifouling paints by cultivated scallops Pecten maximus and pacific oyster Crassostrea gigas, I.C.E.S., CM, 1986/F : 11-18.

14. Evans D.W., Laughlin R.B. Accumulation of bis tributyltin oxide by the mud crab Rhithropanopeus harrisii, Chemosphere, 1984, 13(1) : 213-219.
15. Short J.W., Thrower F.P. Accumulation of butyltin in muscle tissue of chinook salmon reared in sea pens treated with tri-n-butyltin, Ocean's 86 Conference Proceedings. Organotin symposium, 1986, 4 : 11771181.

16. Davies I.M., Mc Kie J.C. Accumulation of total tin and tributyltin in muscle tissue of farmed atlantic salmon, Mar. Pollut. Bull., 1987, 18, 7 : 405-407.

17. Maguire R.J., Tkacz R.J., Chau Y.K., Bengert G.A., Wong P.T.S. Occurrence of organotin coumpounds in water and sediments in Canada, Chemosphere, 1986, $15: 253-60$.

18. Ishizaka T., Nemoto S., Sasaki K., Suzuki, T., Saito Y., Simultaneous determination of tri-n-butyltin, di-n-butyltin, and triphenyltin compounds in marine products, $\mathbf{J}$. Agric. Food Chem, 1989, 37 : 1523-31.

19. Schweinfurth H.A., Gunzel P. The tributyltins : mammalian toxicity and risk evaluation for humans, in Proceedings Ocean's 87, I.E.E.E Service Center, 1987 : 1421-29.

20. Watling-Payne A.S., Selwyn M.J. Inhibition and uncoupling of photophosphorylation in isolated chloroplasts by organotin, organomercury and diphenyleneiodonium compounds, Biochem. J., 1974, 142 : 65-72.

21. Chow S.C., Kass G.E.N., Mc Cabe M.J., Orrenius S., Tributyltin increases cytosolic free $\mathrm{Ca}++$ concentrations in thymocytes by mobilizing intra-cellular $\mathrm{Ca}++$, activating a Ca++ entry pathway, and inhibiting $\mathrm{Ca}++$ efflux, Arch. Biochem. Biophys., 1992, 298, 143-49.

22. Soukry M.M. Equilibrium study of tributyltin(IV) complexes with amino acids and related compounds, Bull. soc. Chim. Fr., 1993, $130: 117-121$.

23. Oehlmann J., Bettin C. TBT-induced imposex and the role of steroids in marine snails, Malacov. Rev. Suppl., 1994, $6: 157-65$.

24. Abalos M., Bayona J-M., Compano R., Granados M., Leal C., Prat M-D. Analytical procedures for the determination of organotin coumpounds in sediment and biota : a critical review, J. Chromatogr. A, 1997, $788: 1-49$.

25. Alzieu C., Sanjuan J., Deltreil, J.P., Borel M. Tin contamination in Arcachon Bay : effects on oyster shell anomalies, Mar. Pollut. Bull., 1986, 17, 11 : 494-498.

26. Claisse D., Alzieu C. Copper contamination as a result of antifouling paint regulations ? Mar. Pollut. Bull., 1993, $26,7: 395-397$. 